\title{
Chapter 21
}

\section{An Error-Control Decision Support System for SWMM}

\author{
W. Robert C. James \\ Computational Hydraulics Int. \\ 36 Stuart Street \\ Guelph, Ontario, Canada N1E 4S5

\section{William James} \\ School of Engineering \\ University of Guelph \\ Guelph, Ontario, Canada N1G 2W1
}

The current version (4.3) of the error-control decision support system, PCSWMM, was developed by the authors for the Microsoft Windows environment, and released in October, 1994. PCSWMM functions as a shell for the US EPA's stormwater management model, SWMM. It simplifies the running of SWMM: provides error-free file management; color plotting of event or continuous SWMM output; advanced sensitivity, calibration and error analysis tools; and field data management. Thus PCSWMM reduces the probability of user error by simplifying normal modeling procedures. This chapter describes the error control features of PCSWMM, especially file management; sensitivity, calibration and error analysis (SCEA) approach; and discusses the future

James, R. and W. James. 1995. "An Error-Control Decision Support System for SWMM." Journal of Water Management Modeling R183-21. doi: 10.14796/JWMM.R183-21.

(C) CHI 1995 www.chijournal.org ISSN: 2292-6062 (Formerly in Modern Methods for Modeling the Management of Stormwater Impacts. ISBN: 0-9697422-4-X) 
of the program as a base for in-house and third-party SWMM enhancements.

\subsection{Introduction}

The US EPA's SWMM models the quantity and quality of urban stormwater runoff. It is a powerful, user-unfriendly program which has seen wide use in North America in the last decade, due in part to its proven reliability, and in part to being public-domain software (i.e. distributed freely by the US EPA). Unfortunately, this lack of commercial revenue has resulted in a program which is error-prone, and the process of learning the program and developing a working model can be fairly lengthy.

The main purpose of PCSWMM is to reduce the probability of user-errors by reducing the complexity of SWMM. File management, datafile linking and interfacing, and especially sensitivity analysis are made faster and simpler, thus controlling errors. The graphical-userinterface has been designed to be fast, flexible, and extremely userfriendly: all commands and procedures can be executed by simple mouse operations (clicks and drag-\&-drops). PCSWMM is compatible with compiled SWMM4.3 from either the US EPA or Oregon State University and works in conjunction with the RAIN, RUNOFF, TRANSPORT, EXTRAN, and STORAGE/TREATMENT modules of SWMM. The program is also designed for facilitating the addition of new tools for SWMM, as discussed in section 21.4. The methodologies for achieving error control, as well as some other features of the program, are described below.

\subsubsection{File Management}

An advanced file management methodology has been developed to ease the organization, connectivity and manipulation of the many files generated and used by SWMM. To give an example of SWMM file complexity, a typical SWMM model might include about ten modules (input data files), as well as meteorological data files. This generates another ten output files, ten interface files, approximately 50 scratch file designations (ranging between three and eight per input data file), ten plot files (ASCII text versions of interface files for spreadsheet plotting), and the requisite batch files for running SWMM. This might result in approximately 100 files. When testing an array of best management 
practices (BMP's), say 50 or more, the number of files involved can be in the thousands. Note that this number does not include the additional thousands of files required for sensitivity analysis (see section 21.2).

In PCSWMM, the above scenario (not including the BMP arrays) would be represented by only 10 file icons, arranged on the screen in a project window shown in Figure 21.1. PCSWMM file icons represent groups of files associated with an input data file (i.e. output, interface, and plot files, as well as sensitivity and calibration files). These file icons are data-aware, and depict the types of input data files they represent (i.e. the SWMM module called). File icons are organized into projects, and can be dragged and dropped to new locations or projects, connected to other file icons, and dropped on the various tools of PCSWMM. To reduce complexity, and thus error, all file extensions are handled automatically by PCSWMM. Data file structures are simplified by eliminating the need for the conventional SWMM EXECUTIVE module command lines from all input data files. Figure 21.2 shows the arcane, non-intuitive input required by the executive module. Instead, data file connectivity is displayed graphically in the project windows, and can be modified at any

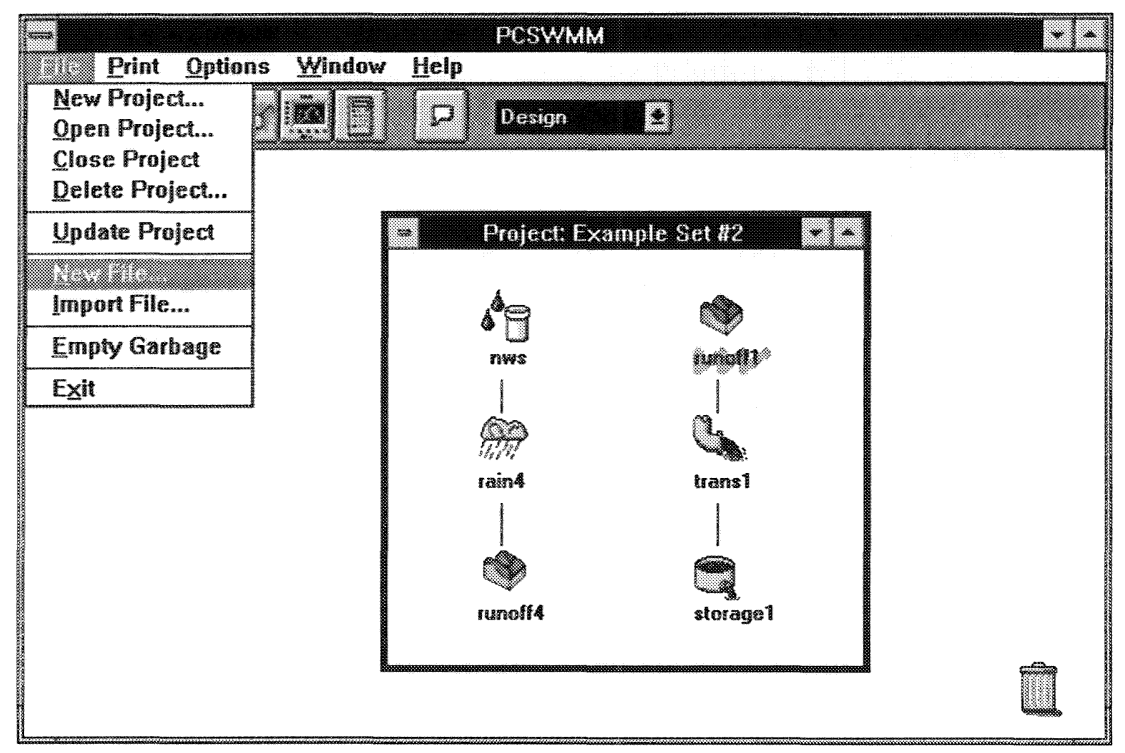

Figure 21.1

PCSWMM graphical-user-interface showing linked file icons arranged in a project window. 


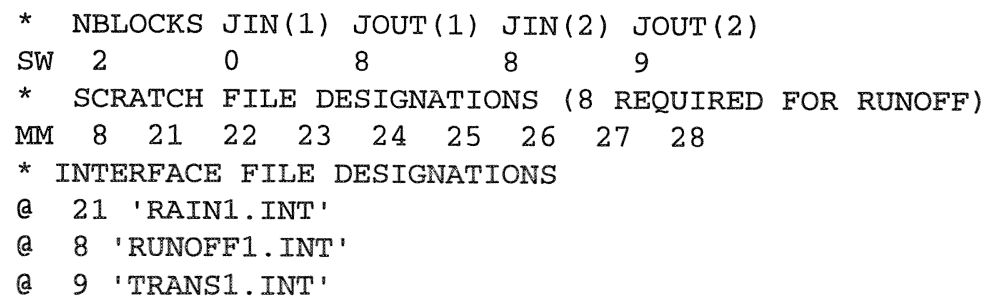

Figure 21.2

Conventional SWMM executive module command lines.

point with a simple mouse click. This reduces complexity and error by eliminating the confusion and problems associated with misplaced interface files, wrong scratch file designations, and long text-oriented commands and input.

Other features of error-free file management include project export/import. Information storage/retrieval is provided for each project and file icon. Existing SWMM files can be imported into PCSWMM, or new data files can be created from user-specified template files. Projects can be exported and imported as a single file for ease of data transfer between PCSWMM computer stations.

\subsubsection{Editing}

PCSWMM works in conjunction with the user's own preferred text editor (Windows or DOS based), so that commands for generating or updating data files are familiar. PCSWMM can be linked to any editor, and the editor can be easily changed on the fly, with the user selecting from a list of common editors, or specifying his/her own. Output files can also be viewed with the selected text editor.

\subsubsection{Running SWMM}

SWMM runs are executed by simply dragging-and-dropping the desired file icon onto the SWMM Run button. The SWMM engine can be either run in a window or full-screen, and changed on the fly. The user can specify the type of SWMM output generated in an options dialog box. This approach avoids the need to generate batch files and/or type in long text command lines. 


\subsubsection{Plotting}

Where users of conventional SWMM must organize SWMM output for visualization into spreadsheet or graphing programs manually (steps that increase the risk of error), PCSWMM instantly generates color hydrographs/pollutographs for the RUNOFF, TRANSPORT, EXTRAN and STORAGE/TREATMENT modules of SWMM for any combination of pipes, channels and inlets. Another advantage of PCSWMM is that continuous simulation output is plotted with over 100,000 data points per plot (spreadsheets are typically limited to 8,000 or so data points). Longterm time series can be scrolled in PCSWMM to identify design events, etc., allowing the user to quickly identify errors in the model data. X, Y1, Y2 plot coordinates are displayed in a variety of units for quick determination of plot statistics (peak flow, time-to-peak, minimum suspended solids conc., etc.).

The object-orientated nature of all PCSWMM plots (including sensitivity and calibration plots) allows for multiple plot windows to be opened, and independently-sized from thumbnails to full screen at any screen resolution. This approach allows great flexibility in combining information presentable at any instant.

\subsubsection{Sensitivity, Calibration and Error Analysis}

The sensitivity approach used in PCSWMM is explained in section 21.2, and the calibration and error analysis is covered in section 21.3.

\subsubsection{Printing}

All plots generated by PCSWMM can be output to any printer supported by Microsoft Windows, in either black-and-white, or color. In addition, data file connectivity schematics can also be printed, thus further reducing user-error.

\subsubsection{Help}

PCSWMM comes with an extensive, online, hypertext help file, which provides a variety of information. Step-by-step instructions are available for all PCSWMM procedures. Hypergraphic representations of 
all PCSWMM screens are included, on which the user can click on various objects to reveal detailed information. Known trouble spots are indexed for quick explanations. Exerpts from Stormwater Management Modeling: A Conceptual Workbook (James, 1992) on sensitivity, calibration and error analysis, are included in the onscreen help file, complete with detailed figures. All help file topics can be printed out. Help and hints are also provided in the PCSWMM program itself by means of an information bar which can be toggled on and off.

\subsubsection{Requirements}

PCSWMM runs on any computer running Windows, requiring 570 kilobytes of free conventional RAM under Windows (needed for the SWMM engine). However, due to the compute-intensive nature of SWMM, and the highly graphical nature of PCSWMM, a 486 or faster computer with high-resolution monitor is recommended.

\subsection{Sensitivity Analysis}

The purpose of sensitivity analysis is to determine which processes control the computed response for the study problem at hand. This is important not only for optimizing the data collection effort, but for parameter optimization (calibration: see section 21.2). Sensitivity analysis must be performed for each individual design effort, because the results vary widely for each application and for different weather events. Once the sensitive processes are identified, the process of model calibration can begin. Unfortunately, a thorough sensitivity analysis can be massively complex. A three point sensitivity analysis (using an expected, low and high parameter estimate) on, say 94 parameters, with 15 objective functions, and 11 input functions (wet and dry weather events) on a single RUNOFF file would require $10^{3}$ to $10^{4}$ files:

$$
N F I L E=[(P A \times 2)+1] \times I N P \times N F I L E R U N
$$

where:

$$
\begin{aligned}
\text { NFILE } & =\text { number of } \mathrm{I} / \mathrm{O} \text { files required for sensitivity analysis } \\
P A & =\text { number of parameters } \\
I N P & =\text { number of input functions }
\end{aligned}
$$




$$
\begin{aligned}
\text { NFILERUN = } & \text { number of files generated/required per SWMM } \\
& \text { run (in this case, two: one input data file and one } \\
& \text { output file) }
\end{aligned}
$$

In this case NFILE $=4,158$ files. If there were more than one RUNOFF module involved, this number of files would be multiplied by the number of RUNOFF module calls. To add to this complexity, all fifteen objective functions must be located in each of the 2,079 output files and collated in a spreadsheet or similar program for analysis. Clearly, the effort required becomes excessive and the potential for error is substantial. On the other hand, PCSWMM can handle the above scenario, generating at a single mouse click, a single sensitivity file for each input function. PCSWMM performs an automatic sensitivity analysis on any combination of 94 different hydrological parameters in the RUNOFF module of SWMM. Parameter uncertainties, input function (both wet and dry event, in either step hyetograph form of varied duration/intensities, or user-specified), and response function location are input by the user. Eleven stephyetograph input functions have the default values suggested by James (1994a) and listed in Table 21.1. They can also be modified by the user at any time. Datafile modifications and sensitivity runs are executed by PCSWMM and output results collated in a file. Results are displayed for

Table 21.1

Default step hyetograph input functions in PCSWMM

\begin{tabular}{|l|c|c|}
\hline Washoff Input Function & Duration (min) & Intensity (mm/hr) \\
\hline Short duration, high intensity (SDHI) & 20 & 76.0 \\
Short duration, medium intensity (SDMI) & 20 & 10.0 \\
Short duration, low intensity (SDLI) & 20 & 2.5 \\
Medium duration, high intensity (MDHI) & 60 & 25.5 \\
Medium duration, medium intensity (MDMI) & 60 & 7.5 \\
Medium duration, low intensity (MDLI) & 60 & 2.5 \\
Long duration, high intensity (LDHI) & 600 & 5.0 \\
Long duration, medium intensity (LDMI) & 600 & 2.5 \\
Long duration, low intensity (LDLI) & 600 & 2.5 \\
\hline Buildup Input Function & Duration (days) & - \\
\hline Short duration, zero intensity (SDZI) & 1 & -- \\
Long duration, zero intensity (LDZI) & 10 & \\
\hline
\end{tabular}


a variety of quantitative and qualitative objective functions in plots of nonlinear normalized sensitivity gradients, or ranked mean normalized sensitivity gradients.

The approach taken in PCSWMM consists of running the original data file of expected parameters with a specified input function (step hyetograph) to generate an expected value for each objective function specified. The data file is then further run twice for each parameter selected for sensitivity analysis. The first run for each parameter uses the data file which has been modified to include the low estimate of that parameter. The second run gives the response function and its objective function for the high estimate of the parameter. As this process is repeated for every parameter, a sensitivity analysis on, say, 19 parameters would require $39 \mathrm{SWMM}$ runs $(19 \times 2+1)$ for each input function chosen.

In a recent calibration effort of the Redhill Creek drainage basin model in Hamilton, Ontario (James 1994b), a sensitivity analysis was performed on the water quantity parameters in the model, a single RUNOFF call. Nineteen different hydrologic parameters were identified as the water quantity parameters for sensitivity analysis, so that these nineteen arrayed parameters for the subcatchments and pipes/channels effectively produced several hundred parameters to be changed. In this application, each of the nineteen parameter arrays were varied by plus and minus five percent $(5 \%)$ of their original value, however PCSWMM allows parameter uncertainties to be set individually. Eight input functions were used to obtain the parameter sensitivities for a range of weather conditions. This gave a total of 312 SWMM runs necessary to generate the required sensitivity analysis. PCSWMM performed all 312 runs, collated the data, evaluated the sensitivity and generated the resulting plots in 24 minutes (running on a $486 \mathrm{dx} 2-66$ computer). The results of the sensitivity analysis consists of sixteen plots: a plot of peak flow and a plot of total flow for each of the eight input functions. Figure 21.3 gives an example of a ranked mean sensitivity gradient plot. To readers familiar with SWMM, it will be obvious that this represents a considerable reduction in complexity and concomitant time savings over a conventional manual sensitivity analysis. The likelihood of user errors is thereby reduced. 


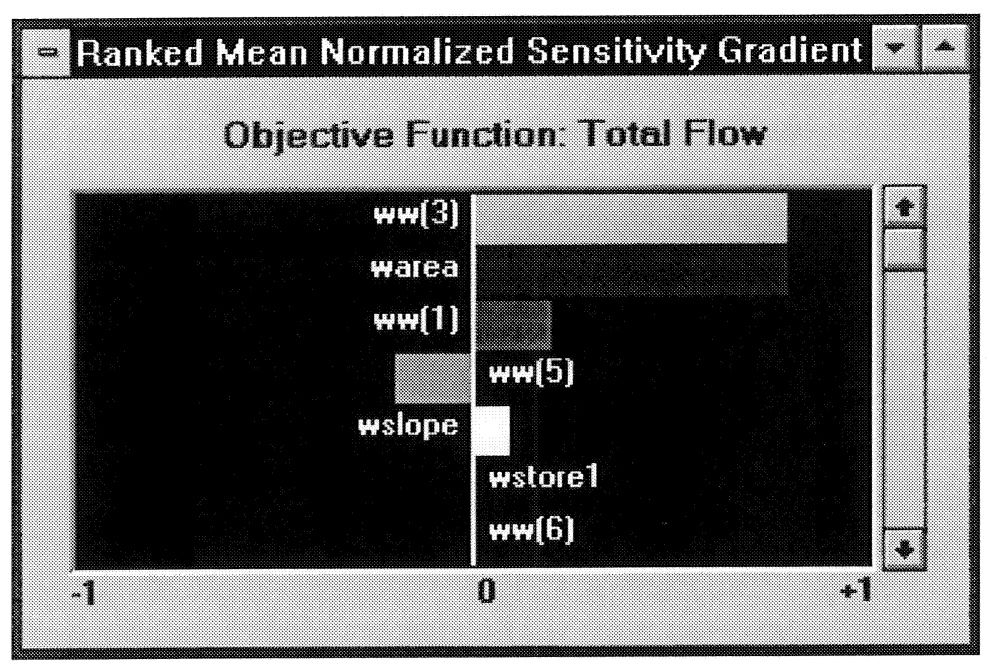

Figure 21.3

Ranked mean normalized sensitivity gradient plot.

\subsection{Calibration and Error Analysis}

Finding an optimal fit of a model's computed output to a single observed event is a trivial task for a model with an input data file containing hundreds of parameters, such as a typical SWMM application. Of course, this should not be misconstrued as model calibration. An infinite set of parameters could form a model calibrated against a single event, and as such is not likely to achieve the same accuracy for other inputs (i.e. other rainfall events, or what-if scenarios). In general, for calibration, the model must use as many observed rainfall events as there are parameters to be calibrated. These observed events should cover the entire spectrum of possible events (i.e. varied durations, intensities and hyetograph shapes). This variation of rainfall events is necessary due to the corresponding variation in parameter sensitivity. For example, infiltration parameters have the greatest effect in short-duration, mediumintensity rainfall events and relatively little effect in short-duration, lowintensity rainfall, where all precipitation infiltrates. They also have little effect in high-intensity long-duration rainfall, where the final infiltration rates are greatly exceeded and most precipitation is converted to runoff. 
However, calibration is rarely performed with such care. Part of the reason for poor calibration lies in the limited observed data usually available for the desired catchment. A more important reason, assuming that sufficient observed data does exist and can be used, is that manual calibration can be laborious, error-prone and thus costly. Calibrating 30 parameters (after the 61 sensitivity analysis runs) requires $30 \mathrm{SWMM}$ runs, with the various objective functions located in the output and plotted using a spreadsheet or plotting program. Computer automation of this process can reduce the probability of user error as well as the modeling cost, by reducing the time required.

The PCSWMM calibration tool illustrated here eases the calibration of models developed with SWMM4.3 by providing observed versus computed (or simulated) plots with error analysis. This section describes the calibration tool and approach. PCSWMM provides tools for generating onscreen calibration plots for an almost unlimited number of userspecified objective functions (in excess of 250), with a maximum of 20 observed and simulated data pairs, entered by the user, for each objective function selected. The calibration plotting window displays a plot of the observed vs. simulated objective functions. In this plot, a 45 degree line is drawn from the lower left corner to the upper right corner. This line represents the desired location of all data for a perfectly calibrated model. The degree of variance of the data from this line represents the error for the selected objective function. The error is represented by two error functions, which are automatically calculated for each objective function and displayed in the upper left corner of the calibration plot.

There are numerous common error functions used to evaluate the performance of computed response functions and/or objective functions. A list of 24 of these error functions that have been used in previous model calibration efforts is given by James (1994). The two error values currently calculated by the calibration tool are:

1. the standard error measure (E1):

$$
E 1=\sqrt{\frac{\sum_{i=1}^{n}\left(\operatorname{sim}_{i}-o b s_{i}\right)^{2}}{n}}
$$


2. the normalized standard error measure (E2):

$$
E 2=\sqrt{\frac{\sum_{i=1}^{n}\left(\frac{s i m_{i}-o b s_{i}}{o b s_{i}}\right)^{2}}{n}}
$$

It is difficult in error analysis to find a universal measure of error to summarize the variance in the data in a meaningful way for all inferences. In the case of the two error measurements adopted, E1 tends to be dominated by variance at the upper limits of the objective function, whereas the normalized error term, E2, is dominated by variances at the low end of the objective function range. Because of this, other error measurements may be more desirable for specific calibration objectives, and the authors can provide these on request. The calibration tool, while not as automatic as the sensitivity analysis tool (the user is required to manually enter the observed/computer data pairs), provides a quick view of model certainty.

\subsection{Future Directions}

The next step for PCSWMM is to create an extremely flexible and adaptible kernel program that works with any SWMM application to dynamically display calibration tuning and error. We hope, by incorporating linear error analysis, to create a DSS environment which can seamlessly interact with SWMM applications in Windows to provide multi-valued (fuzzy) plots, and radio-tuning for calibration.

We are currently developing an open structure for facilitating PCSWMM Enhancements. Enhancements are small external programs, compiled in any language, and linked to PCSWMM through either Object Linking and Embedding (OLE 2.0) support or Dynamic Data Exchange (DDE) format. Even application languages such as macros or Visual Basic for Applications can be linked in this way. This open structure provides a simple and effective pathway for in-house developments to be integrated into a customized, comprehensive modeling environment. For example, where a modeling contract specifies a standard format for graphing calibration and error analysis data, a macro could be written in a spreadsheet program to display this graph format, save it, and, with a couple 
simple steps, link it to the calibration tool in PCSWMM. As the calibration data changes during the project, or even between projects, the standard graphical output is a button-click away.

In our opinion, an open structure for PCSWMM will facilitate the inclusion of graduate work. Many Masters and Doctorate theses in the area of water quality and quantity modeling include useful core code, eg. time-series management (Gregory, 1995) three-generation, continuous modeling tools written in C (Kuch, 1995), storm tracking by small radar (Abdul-Kader, 1995) and others. These programs often never see further use, bogged down by eclectic interfaces and shaky support code (often extensive code that is required for file handling and user interfacing - but not for graduation). This is to be expected, as they are written by environmental and water resource engineers for academic purposes, and not by professional programmers for commercial markets. By providing the DDE and OLE 2.0 compliant core program (i.e. PCSWMM) these program codes can be linked seamlessly and with little effort. Buttons and menu commands can be embedded in PCSWMM that send task information and data to these programs, avoiding the need to develop separate interfaces and file handlers. This strategy can also be adopted by PCSWMM users and/or third parties as graduate code is often readily available.

\subsection{Conclusions}

We envision a shareware community consisting of consulting, municipal and post-secondary educational water quality and quantity modelers, with an ever-increasing number of enhancements being passed around, of which PCSWMM simply plays a part. Any computer equipped with a modem or internet access will be able to download these programs from Guelph and/or other locations (i.e. GLIN, CIESIN, etc).

Most modern programming languages and application software packages support the DDE and OLE 2.0 standards, and with the proliferation of powerful, high level languages, such as Visual Basic and Galaxy, and wide adoption of the open structure approach to software development, we will see a rapidly-expanding base of shareware in the water quality and quantity modeling arena. 


\section{References}

Abdul-Kader, S., 1994, Storm Tracking by Small-scale Radar, Unpublished proposal for graduate research - copy available from W. James, University of Guelph, Guelph, Ontario.

Gregory, M., 1995, Time-Series Management, Unpublished proposal for graduate research - copy available from W. James, University of Guelph, Guelph, Ontario.

Henry, D., 1983, Hamilton Stormwater Management Study, MEng. Thesis, McMaster University, Hamilton, Ontario, 144pp.

Huber, W.C. and R.E. Dickinson, 1988, Stormwater Management Model Version 4: User's Manual, U.S. Environmental Protection Agency, Athens, Georgia, 569pp.

James, W., 1994a, Rules for Responsible Modeling, Computational Hydraulics Int., Guelph, Ontario, 144pp.

James, W. and W.R.C. James, In Press, PCSWMM - a public domain, graphics oriented shell for easing the design-use of SWMM4. Runoff Quantity and Quality Model Group Conference. ASCE, Reno, Nevada, Nov. 8-9, 1993, 6pp.

James, W.R.C., 1994b, Calibration Tools for Stormwater Management Modelling, Undergraduate final design report for Environmental Engineering, University of Guelph, Guelph, Ontario, 70pp.

Kuch, A., 1994, Three-Generation, Continuous Modelling Tools, Unpublished proposal for graduate research - copy available from W. James, University of Guelph, Guelph, Ontario.

Robinson, M.A. and W. James 1984, Continuous SWMM3 Modeling of Summer Stormwater Quality in the Redhill Creek, Report No. 111, CHI Publications Guelph, Ontario, 100pp. 
